\title{
Virulence Genotype and Clinical Influence of the Type VI Secretion System in Klebsiella Pneumoniae Isolates Causing Bloodstream Infections
}

\section{Yin Zhang}

First Affiliated Hospital of Anhui Medical University

Ying Huang ( $\nabla$ huangying_ah2007@sina.com )

The First Affiliated Hospital of Anhui Medical University

\section{Research}

Keywords: hypervirulent Klebsiella pneumoniae, bloodstream infection, virulence, antibacterial, bacterial competition, Type VI Secretion System

Posted Date: June 25th, 2021

DOI: https://doi.org/10.21203/rs.3.rs-651386/v1

License: (c) (i) This work is licensed under a Creative Commons Attribution 4.0 International License.

Read Full License 


\section{Abstract}

\section{Background}

Klebsiella pneumoniae (K. pneumoniae) causes bloodstream infection (BSI), which is responsible for a high rate of morbidity and mortality among different populations. In mainland China, data on the incidence and features of the T6SS gene cluster in K. pneumoniae is currently scarce. As a result, we conducted a prospective investigation to determine the involvement of the type VI secretion system (T6SS) in K. pneumoniae pathogenicity and antibiotic resistance.

Method

In this retrospective analysis, we enrolled 119 individuals who had been diagnosed with K. pneumoniae bloodstream infection and acquired demographic and clinical data from their medical records. The virulence genes rmpA, rmpA2, aerobactin, iroB, hcp, vgrG, and icmF were tested for $\mathrm{K} 1$ and $\mathrm{K} 2$, antimicrobial susceptibility. T6SS positive strains $(\mathrm{N}=20)$ were identified as having icmF, vgrG, and hcp, while T6SS negative strains ( $\mathrm{N}=99)$ did not manifest the same. In this study, hvKP was defined as rmpA and aerobactin positivity. Five T6SS+ and five T6SS- isolates were chosen for the competition, serum resistance, and biofilm formation experiments to further gain insights regarding the microbiological properties of T6SS+K. pneumoniae isolates.

Result

Among 119 isolates obtained from patients with BSIs, 20 (16.8\%) were T6SS positive K. pneumoniae. T6SS positive strains had four virulence genes and a greater K1 capsular serotypes rate than T6SS negative bacteria. Among hvKP isolates, the T6SS positive rate was substantially greater than the T6SS negative rate $(P=0.001)$. T6SS-positive $K$. pneumoniae strains had a lower rate of antimicrobial resistance in comparison to T6SS-negative bacteria. The 30-day mortality in all patients was $23.1 \%$, and $66.7 \%$ (26 patients) of them died in the first 7 days of bacteremia onset. The T6SS genotype determined no significant differences in early (7-day) mortality. On the other hand, late mortality among patients with T6SS+ isolates were $10 \%$ compared with $37.4 \%$ among patients infected by T6SS- strains ( $\mathrm{P}=$ 0.01). In comparison to T6SS-negative isolates, $K$. pneumoniae isolates with T6SS-positive might outcompete Escherichia coli. T6SS+ isolates, on the other hand, did not show stronger biofilm-forming activity or a greater survival rate in presence of normal human serum in comparison to T6SS- isolates.

\section{Conclusion}

Individuals with BSIs were more likely to have T6SS-positive $K$. pneumoniae. T6SS+ K. pneumoniae strains appeared to be extremely virulent. In T6SS-containing $K$. pneumoniae, the system may play a major role in bacterial competition.

\section{Introduction}


Klebsiella pneumoniae (K. pneumoniae), an opportunistic infecting microbe, frequently causes pneumonia, bacteremia, liver abscess, and urinary tract infection [1-2]. Multidrug-resistant strains of $K$. pneumoniae are common, thus leading to failure of antibiotics therapy against different infections. Although most medicines are effective against nonclassical hypervirulent $K$. pneumoniae strains, they can induce meddlesome infections in both immunocompromised and healthy patients within the community [3]. New $K$. pneumoniae strains with hypervirulence and multidrug resistance have recently been discovered in China, raising worries about $K$. pneumoniae infections [4-6].

The bacterial Type VI Secretion System (T6SS) is a membrane-attached contractile phage tail that is physically and mechanistically similar to a membrane-linked intracellular contractile phage tail [7-8]. T6SS spike and tube elements, as well as anti-bacterial and anti-eukaryotic effectors, are propelled out of predatory T6SS + cells and into target cells by a fast conformational shift in the structural framework of a sheath protein complex, according to recent research [9]. In an ATP-dependent mechanism, the constricted organelle is then recycled. The T6SS has the potential of targeting bacteria co-infecting a mammalian host in addition to eukaryotic cells, thus revealing its importance for disease and infection as well as bacterial survival in environmental ecosystems.

A functioning T6SS secretes hemolysin-coregulated protein ( $\mathrm{Hcp}$ ) into the extracellular environment [1011]. Owing to their reciprocal needs for secretion, $\mathrm{Hcp}$ and another widely reported extracellular protein (or exoprotein), valine-glycine repeat protein $\mathrm{G}(\mathrm{VgrG})$, are devoid of a detectable Tat or Sec-dependent signal peptide and are a fundamental element of the secretion apparatus [12]. In vitro, Hcp creates a hexamer ring having a 40-pore internal pore and has the ability to be stacked as a tube-like structural form.

Surprisingly, the structural similarity and sequence of Hcp and VgrG to T4 bacteriophage spike gp5/gp27 and tail tube gp19 has led to suggesting an Hcp-VgrG phage tail-like structure [13].

ATPases or proton motive forces are commonly used in bacterial secretion systems to energize secretion apparatus assembly and/or substrate transfer. A pair of proteins whose ATPase activity or nucleotidebinding sites are already known and could be used as energizers in T6SSs [14]. TssH (ClpV), a AAA+-type ATPase, facilitates ATP hydrolysis-mediated remodeling of cogwheel-like TssB/TssC (VipA/VipB) tubules, required for $\mathrm{Hcp}$ secretion [15-16]. TssM belongs to the intracellular multiplication protein $\mathrm{F}$ (IcmF) family and is a T6SS inner membrane protein. TssM's NTPase activity and role in T6SS remain unknown, apart from the persistence of its Walker A nucleotide-binding motif [17]. TssM, belonging to the IcmF family of proteins, has ATPase activity and induces the secretion of type $\mathrm{VI}$, according to one study [18].

T6SSs are implicated in anti-virulence or virulence, cytotoxicity to prokaryotic hosts or eukaryotic, and biofilm formation implying that this secretion system has a wide range of functions [19-21]. The T6SS gene cluster of Ralstonia solanacearum, for example, plays a key role not only in plant pathogenicity but also in motility and biofilm generation. MFE01tssC, a mutant of Pseudomonas fluorescens MFE01 that lacks one of the primary constituent genes, was not able to form its own biofilm [20]. The T6SS has also been linked to biofilm development in Burkholderia cenocepacia [22]. This mechanism is also engaged in 
cell-to-cell communication and signaling. The T6SS is used by Vibrio cholerae to cause variation in the cellular activity of the host that lowers the population of competing bacteria.

The goal of this study was to look at the involvement of the type VI secretion system (T6SS) in $K$. pneumoniae pathogenicity and antibiotic resistance. T6SS-producing and non-T6SS-producing $K$. pneumoniae clinical isolates were examined in terms of antimicrobial resistance and virulence characteristics. In vitro tests, for instance, competition, biofilm formation, and serum resistance assays were used to evaluate T6SS function.

\section{Materials And Methods}

\subsection{Study designs and data collection}

The First Affiliated Hospital of Anhui Medical University, a 3000-bed tertiary-level healthcare facility in Hefei, China, hosted this single-center prospective cohort study from July 2019 to January 2021. The current investigation was duly approved by the human ethics council of Anhui Medical University's First Affiliated Hospital. As no personal information was used in the study, patients were not required to provide a declaration of permission for submission.

The BacT/ALERT 3D system was used for culturing K. pneumoniae BSI, which was isolated from one or more sets of aseptically acquired blood culture bottles (Becton-Dickinson, Sparks, MD, USA). Blood was cultured in the microbiology lab employing a BacT/ALERT 3D system (Becton-Dickinson, Sparks, MD, USA). Matrix-associated laser desorption ionization-time of flight mass spectrometry (Vitek MS, Biomerieux, France) was utilized for the recognition of all isolates. Following the Clinical and Laboratory Standards Institute's recommendations, antibiotic susceptibility testing was done making use of the VITEK 2 (Card number: AST-GN13) system or the methods of Kirby-Bauer Disk Diffusion (Oxoid, UK) (CLSI). Escherichia coli ATCC25922 and K. pneumoniae ATCC700603 were employed as quality control isolates.

\subsection{Clinical Data and Definitions}

Medical data were used to acquire clinical information on $119 \mathrm{KP}$-infected patients. Immunosuppression was referred to as primary immunodeficiency disorder and/or regular use of high-dose steroid therapy (prednisolone $10 \mathrm{mg} /$ daily or comparable dose) for more than half a month, and/or immunosuppressive chemotherapy within the previous two months. Neutropenia was classified as a neutrophil count of fewer than 500 per liter. When symptoms and positive blood culture were gathered more than 48 hours following hospital admission, it was considered a nosocomial infection. MDR (multi-drug resistance) is referred to as acquired non-susceptibility to at least one antimicrobial entity from three or more groups. The length of duration between the automated system's alert signal and the start of blood incubation and was assessed as time to positive (TTP). In case of the presence of more than one positive sample, we used the shortest TTP. Appropriate antibiotic therapy meant receiving a least one intravenous active antimicrobial treatment based on susceptibility results within 24 hours of collecting blood samples and 
before susceptibility findings were available; In any other circumstance, unsuitable antimicrobial therapy was defined [24].

\subsection{Detection of virulence genes, capsular serotypes, and T6SS genes}

A commercial DNA extraction kit was used to extract DNA from K. pneumoniae isolates (Sangon Biotech, Shanghai, China). From an overnight culture, $1 \mathrm{~mL}$ of bacterial suspension matched comparable to 0.5 McFarland was made and centrifuged. DNA was extracted from the pellet according to the kit's

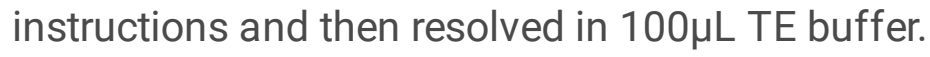

PCR-based detection of biomarker genes employed $12.5 \mu$ of $2 \times$ Spark Taq PCR Master Mix (Solely Bio,Shandong $\square$ China), $1 \mu \mathrm{l}$ of forward primer, $1 \mu \mathrm{l}$ of reverse primer $(10 \mathrm{pmol} / \mu \mathrm{l}$ primer stock $), 1 \mu \mathrm{l}$ of genomic DNA, and $9.5 \mu$ of water. PCR was performed using a Biometra thermocycler with the cycling conditions mentioned as follows: First step: $94.0^{\circ} \mathrm{C}$ for $3 \mathrm{~min}$; Second step: $94.0^{\circ} \mathrm{C}$ for $30 \mathrm{~s}$; Third step: primer-specific annealing temperature for $30 \mathrm{~s}$; Forth step: $72^{\circ} \mathrm{C}$ for $1 \mathrm{~min}$; Fifth step: repeating steps 2-4 for 35 cycles; Sixth step: $72.0^{\circ} \mathrm{C}$ for $10 \mathrm{~min}$; Seventh step: holding at $4^{\circ} \mathrm{C} .1 \%$ agarose gel was used to resolve the amplified products. Additional file 1: Table S1 lists all the primers employed. 
Table S1

List of primers used in this study

\begin{tabular}{|c|c|c|}
\hline Primer name & DNA sequence $\left(5^{\prime} \rightarrow 3^{\prime}\right)$ & Amplicon size (bp) \\
\hline \multicolumn{3}{|c|}{ Capsular serotypes } \\
\hline \multirow[t]{2}{*}{ K1 } & F: GTAGGTATTGCAAGCCATGC & 1283 \\
\hline & R: GCCCAGGTTAATGAATCCGT & \\
\hline \multirow[t]{2}{*}{ K2 } & F: GGAGCCATTTGAATTCGGTG & 641 \\
\hline & R: TCCCTAGCACTGGCTTAAGT & \\
\hline \multirow[t]{2}{*}{ K5 } & F: GCCACCTCTAAGCATATAGC & 280 \\
\hline & R: CGCACCAGTAATTCCAACAG & \\
\hline \multirow[t]{2}{*}{ K20 } & F: CCGATTCGGTCAACTAGCTT & 741 \\
\hline & R: GCACCTCTATGAACTTTCAG & \\
\hline \multirow[t]{2}{*}{ K54 } & F: CATTAGCTCAGTGGTTGGCT & 881 \\
\hline & R: GCTTGACAAACACCATAGCAG & \\
\hline \multirow[t]{2}{*}{ K57 } & F: CGACAAATCTCTCCTGACGA & 1037 \\
\hline & R: CGCGACAAACATAACACTCG & \\
\hline \multicolumn{3}{|c|}{ virulence genes } \\
\hline \multirow[t]{2}{*}{ rmpA } & F: ACTGGGCTACCTCTGCTTCA & 516 \\
\hline & R: CTTGCATGAGCCATCTTTCA & \\
\hline \multirow[t]{2}{*}{ rmpA2 } & F: CTTTATGTGCAATAAGGATGTT & 451 \\
\hline & R: CCTCCTGGAGAGTAAGCATT & \\
\hline \multirow[t]{2}{*}{ aerobactin } & F: ACAGGGCAATTGCTTACCTCCT & 556 \\
\hline & R: GCATAGGCGGATACGAACAT & \\
\hline \multirow[t]{2}{*}{ iroB } & F: АТСТСАТСАТСТАСССТССGСТC & 235 \\
\hline & R: GGTTCGCCGTCGTTTTCAA & \\
\hline \multicolumn{3}{|l|}{ T6SS genes } \\
\hline \multirow[t]{2}{*}{$\mathrm{Hcp}$} & F: TCCCGACCGATAACAACACC & 242 \\
\hline & R: GATGTCGTGCATCAGGGGAT & \\
\hline \multirow[t]{2}{*}{ VgrG } & F: TGAGCGTGTTTGTGCGAAAG & 259 \\
\hline & R: TGACGCCCGTAATATCCTGC & \\
\hline
\end{tabular}




\begin{tabular}{|lll|}
\hline Primer name & DNA sequence $\left(\mathbf{5}^{\prime} \rightarrow \mathbf{3}^{\prime}\right)$ & Amplicon size $(\mathbf{b p})$ \\
\hline $\mathrm{IcmF}$ & F: GACCGCTTACGGACAACTGA & 485 \\
& R: CACTCAGCACCCAGTCCATT & \\
\hline
\end{tabular}

\subsection{Biofilm assay}

Biofilm formation tests were carried out as described earlier, with a number of tweaks [25]. The OD600 was used to standardize overnight cultures. The suspension was inoculated into the wells of a polystyrene $96-w e l l$ flat-bottom plate using a $200-\mu \mathrm{L}$ aliquot and left to incubate for 18 hours at $37^{\circ} \mathrm{C}$. The medium was then taken from the plates, and each well was thoroughly washed three times with water. After 30 minutes of air-drying, crystal violet solution $(1 \%, 150 \mu \mathrm{L})$ was applied to individual wells for 15 minutes. The colorant was discarded after staining the adherent cells, and $200 \mu \mathrm{L}$ of distilled water was used to rinse the wells three times in order to eliminate any surplus stain. For 0.5 hours, the plates were air-dried. In $200 \mu \mathrm{L}$ of $100 \%$ ethanol, the dye absorbed by the adhering cells was solubilized. As a negative control, sterile LB was employed. The stained adherent bacteria and control wells had their OD600 values tested. Three separate studies, each with three replicate wells, were carried out. Biofilm production positive was defined as a strain with an absorbance value greater than 2 times that of the blank absorbance.

\subsection{Serum resistance assay}

The serum resistance analyses were carried out as elaborated earlier, with minimal modifications [26]. Dilution 1:100 of overnight bacterial cultures was carried out into fresh LB media $(10 \mathrm{~mL})$ and the diluted specimens were allowed to incubate until an OD600 of 0.5 was achieved. The culture was then rinsed in phosphate-buffered saline (PBS) for $1 \mathrm{~mL}$ followed by resuspension in $1 \mathrm{~mL}$ of PBS. Following that, 100 $\mu \mathrm{L}$ of the bacterial suspension were combined with $300 \mu \mathrm{L}$ of normal human serum (NHS). The suspensions of serum-bacteria were mixed and then set to incubate at $37^{\circ} \mathrm{C}$ for 3 hours. A $100-\mu \mathrm{L}$ aliquot of each culture was obtained prior to and following the 3-h period of incubation, diluted serially, and plated for calculating the serum bactericidal effect. The serum bactericidal effect was calculated using the ratio of serum-bacteria suspension CFUs to bacterial suspension CFUs without NHS. All studies were carried out in triplicate, and the findings are expressed as percent survival.

\subsection{In vitro competition assay}

Strains of the attacker (K. pneumoniae) and prey (E. coli MG1655) were cultured in LB overnight at $37^{\circ} \mathrm{C}$. Each culture was then diluted 1:100 in $10 \mathrm{~mL}$ of LB broth after being standardized to an OD600 of 0.5 . The suspensions of bacteria were mixed in a 2:1 attacker: prey ratio and cultured for 20 hours at $180 \mathrm{rpm}$ and $37^{\circ} \mathrm{C}$. The determination of cells count for each strain was carried out via pouring their serial 10 -fold dilutions onto LB agar plates in the presence or absence of ampicillin $(50 \mathrm{mg} / \mathrm{L})$ [27-28]. An in vitro competitive index was calculated to measure the relative fitness of $K$. pneumoniae isolates against $E$. coli strain $(\mathrm{Cl})$. The $\mathrm{Cl}$ was calculated as the ratio of ampicillin-resistant $K$. pneumoniae to ampicillinsusceptible CFUs E. coli. A total of ten competition experiments were carried out. 


\subsection{Statistical analysis}

In the case of continuous variables, medians and interquartile ranges (IQR) were used, whereas, for categorical variables, relative and absolute frequencies were employed. The Student's t-test or the MannWhitney $U$ test, as well as the 2 test or Fisher's exact test, were used for the comparison of categorical and continuous parameters. The log-rank test was carried out to examine differences between groups, and Kaplan-Meier survival curves were employed for survival analysis. Patients who were not evaluable for crude mortality analysis were those who were discarded for aggressive antimicrobial therapy due to endstage illness. All statistical tests were two-tailed, and statistical significance was referred to as a P-value equivalent to 0.05 . The SPSS statistical package v.24.0 (SPSS Inc., Chicago, IL, USA) was employed to conduct the analyses, and Prism software v.9.0 (GraphPad Inc., La Jolla, CA, USA) was used to create the visualizations.

\section{Results}

\subsection{Study population and patient characteristics}

In the duration of the study, one hundred and thirty inpatients with a positive K. pneumoniae blood culture were included. Eleven of them were disqualified (five cases were newborns, four cases were infected with other bacteria, two cases had incomplete information). As a result, 119 instances were finally examined in this work.

The average age of these individuals was $51.6 \pm 16.9$ years, with men accounting for $69.7 \%(83 / 119)$ of the total. The average duration of the stay in the hospital was $37.9 \pm 45.2$ days. Hypoalbuminemia $(82.3 \%, 98 / 119)$ was the most frequent underlying condition, followed by immunosuppression $(43.7 \%$, $52 / 119)$. Diabetes $(32 / 119,26.9 \%)$ was the most common underlying condition, followed by hypertension and end-stage renal disease $(22 / 119,18.4 \%) .47$ patients (or $39.4 \%)$ were admitted to the ICU. Forty patients (33.6\%) were community-acquired infection. The Charlson Comorbidity Index has a median of $1.8(0-6.00)$. Eighty-three patients $(69.7 \%)$ received suitable empirical antibiotic treatment. MDR microorganisms were found in seventy-eight (65.5\%) of the patients (78/119). The mortality rate at the hospital was $36.1 \%(43 / 119)$. Table 1 provides more information on clinical features.

The features of the positive and negative T6SS groups are depicted in Table 1. Patients in the T6SS negative group had significantly greater 30 -day mortality $(37.4 \%$ vs. $10 \%, P=0.01)$, MDR $(71.7 \%$ vs. 35.0 $\%, P=0.002)$, and immunosuppressive patients ( $48.5 \%$ vs. $20.0 \%, P=0.01)$. T6SS positive patients had higher rates of hypertension than T6SS negative patients $(40.0 \%$ vs $14.1 \%, P=0.007)$. There were no considerable differences in the underlying diseases, community infectious infection, antibiotic administration, or length of hospitalized stay. 
Table 1

Baseline characteristics of patients with klebsiella pneumoniae bloodstream infection

\begin{tabular}{|c|c|c|c|c|}
\hline Patient characteristic & $\begin{array}{l}\text { All } \\
(n=119)\end{array}$ & $\begin{array}{l}\text { T6SS-positive } \\
(n=20)\end{array}$ & $\begin{array}{l}\text { T6SS-negative } \\
(\mathrm{n}=99)\end{array}$ & $\stackrel{p-}{\text { Value }}$ \\
\hline Age, years, mean $\pm S D$ & $51.6 \pm 16.9$ & $60.2 \pm 12.4$ & $49.8 \pm 17.1$ & 0.01 \\
\hline Gender, \% male & $83(69.7)$ & $14(70)$ & $69(69.7)$ & 0.97 \\
\hline CCl,median(IQR) & $1.8(0-6)$ & $1.9(0-4)$ & $1.8(0-6)$ & 0.85 \\
\hline \multicolumn{5}{|l|}{ Underlying diseases, $\mathrm{n}(\%)$} \\
\hline Diabetes mellitus & $32(26.9)$ & $8(40.0)$ & $24(24.2)$ & 0.14 \\
\hline Hypertension & $22(18.4)$ & $8(40.0)$ & $14(14.1)$ & 0.007 \\
\hline $\begin{array}{l}\text { Chronic cardiac } \\
\text { insufficiency }\end{array}$ & $7(5.9)$ & $1(5.0)$ & $6(6.1)$ & 0.85 \\
\hline End-stage renal disease & $22(18.4)$ & $2(10.0)$ & $20(20.2)$ & 0.28 \\
\hline Chronic liver disease & $11(9.2)$ & $1(5.0)$ & $10(10.1)$ & 0.47 \\
\hline Cerebrovascular diseases & $6(5.0)$ & $1(5.0)$ & $5(5.1)$ & 0.99 \\
\hline $\begin{array}{l}\text { Hematological } \\
\text { malignancies }\end{array}$ & $15(12.6)$ & $2(10.0)$ & $13(13.1)$ & 0.7 \\
\hline Solid tumor & $15(12.6)$ & $3(15.0)$ & $12(12.1)$ & 0.72 \\
\hline Transplant & $22(18.4)$ & $1(5.0)$ & $21(21.2)$ & 0.08 \\
\hline \multicolumn{5}{|l|}{ Underlying conditions } \\
\hline Immunosuppression & $52(43.7)$ & $4(20.0)$ & $48(48.5)$ & 0.01 \\
\hline Previous surgery & $41(34.5)$ & $5(25.0)$ & $36(36.4)$ & 0.32 \\
\hline Neutropenia & $22(18.4)$ & $2(10.0)$ & $20(20.2)$ & 0.28 \\
\hline Hypoalbuminemia & $98(82.3)$ & 16(80.0) & $82(82.8)$ & 0.76 \\
\hline ICU stay & $47(39.4)$ & $9(45.0)$ & $38(38.4)$ & 0.58 \\
\hline Community-acquired BSI & $40(33.6)$ & $9(45.0)$ & $31(31.3)$ & 0.23 \\
\hline Healthcare-associated & $79(66.4)$ & $11(55.0)$ & $68(68.7)$ & 0.23 \\
\hline TTP & $14.3 \pm 19.3$ & $10.6 \pm 8.6$ & $15.0 \pm 10.1$ & 0.36 \\
\hline Hospitalization days & $37.9 \pm 45.2$ & $34.6 \pm 38.2$ & $38.6 \pm 46.6$ & 0.71 \\
\hline $\begin{array}{l}\text { Multi-drug resistance } \\
\text { bacteria }\end{array}$ & $78(65.5)$ & $7(35.0)$ & 71(71.7) & 0.002 \\
\hline
\end{tabular}

CCI, Charlson Comorbidity Index; IQR, interquartile range; TTP, Time to positivity. 


\begin{tabular}{|lllll|}
\hline Patient characteristic & $\begin{array}{l}\text { All } \\
(\mathbf{n = 1 1 9 )}\end{array}$ & $\begin{array}{l}\text { T6SS-positive } \\
(\mathbf{n = 2 0 )}\end{array}$ & $\begin{array}{l}\text { T6SS-negative } \\
(\mathbf{n = 9 9 )}\end{array}$ & $\begin{array}{c}\text { P- } \\
\text { Value }\end{array}$ \\
\hline $\begin{array}{l}\text { Initial appropriate } \\
\text { antibiotics }\end{array}$ & $83(69.7)$ & $17(85.0)$ & $66(66.7)$ & 0.10 \\
\hline Outcomes & & & $23(23.2)$ & 0.41 \\
\hline 7 day total mortality rate & $26(21.8)$ & $3(15.0)$ & $37(37.4)$ & 0.01 \\
\hline 30 day total mortality rate & $39(32.8)$ & $2(10.0)$ & $38(38.4)$ & 0.25 \\
\hline In-hospital mortality (n,\%) & $43(36.1)$ & $5(25.0)$ & \\
\hline CCl, Charlson Comorbidity Index; IQR, interquartile range; TTP, Time to positivity. & \\
\hline
\end{tabular}

\subsection{Microbiological characteristics and antimicrobial susceptibility}

Apart from natural resistance to ampicillin, $K$. pneumoniae had the highest rate of resistance towards piperacillin, whereas tigecycline resistance was as low as $0.8 \%$. T6SS-positive $K$. pneumoniae strains also had lower rates of antimicrobial resistance than T6SS-negative bacteria. With the exception of minocycline, tigecycline, and ceftazidime averbactam, practically all antibiotic drugs had significantly greater resistance rates for T6SS negatives than for T6SS positives. $24(20.2 \%)$ of the $119 \mathrm{~K}$. pneumoniae clinical isolates were found to be ESBL-producing. Seventy-eight strains (65.5\%) were found to be multidrug-resistant bacteria (MDR), which were shown to be more prevalent in the T6SS-negative group $(71.7 \%$ vs $35.0 \%, P=0.002)$. Carbapenem-resistant $K$. pneumoniae $(\mathrm{CRKP})$ was also found in lower numbers in the T6SS-positive strain $(20.0 \%$ vs $45.4 \%, P=0.03)$. Table 2 has more information on these findings. 
Table 2

Antimicrobial resistance of T6SS-positive and T6SS-negative $K$ pneumoniae bloodstream isolates

\begin{tabular}{|c|c|c|c|c|}
\hline $\begin{array}{l}\text { Antimicrobial } \\
\text { susceptability,n(\%) }\end{array}$ & $\begin{array}{l}\text { All } \\
(n=119)\end{array}$ & $\begin{array}{l}\text { T6SS-positive } \\
(n=20)\end{array}$ & $\begin{array}{l}\text { T6SS-negative } \\
(n=99)\end{array}$ & $p$ \\
\hline Aztreonam & $65(54.6)$ & $6(30.0)$ & $59(59.6)$ & 0.01 \\
\hline Gentamicin & $58(48.7)$ & $5(25.0)$ & $53(53.5)$ & 0.02 \\
\hline Tobramycin & $45(37.8)$ & $3(15.0)$ & $42(42.4)$ & 0.02 \\
\hline Trimethoprim sulfamethoxazole & $51(42.8)$ & $5(25.0)$ & $46(46.5)$ & 0.07 \\
\hline Amikacin & 38 (31.9) & $3(15.0)$ & $35(35.4)$ & 0.07 \\
\hline Cefepime & $56(47.0)$ & $6(30.0)$ & $50(50.5)$ & 0.09 \\
\hline Ceftriaxone & $75(63.0)$ & $8(40.0)$ & $67(67.7)$ & 0.01 \\
\hline Cefotaxime & $76(63.8)$ & $8(40.0)$ & $68(68.7)$ & 0.015 \\
\hline Ceftazidime & $62(52.1)$ & $6(30.0)$ & $56(56.6)$ & 0.03 \\
\hline Cefoperazone-sulbactam & $57(47.8)$ & $4(20.0)$ & $53(53.5)$ & 0.006 \\
\hline Ciprofloxacin & $68(57.1)$ & $4(20.0)$ & $64(64.6)$ & $\nabla 0.001$ \\
\hline Levofloxacin & $58(48.7)$ & $3(15.0)$ & $55(55.6)$ & 0.001 \\
\hline Imipenem & $50(42.0)$ & $4(20.0)$ & $46(46.5)$ & 0.029 \\
\hline Meropenem & $50(42.0)$ & $4(20.0)$ & $46(46.5)$ & 0.029 \\
\hline Ampicillin-sulbactam & 79 (66.3) & $8(40.0)$ & $71(71.7)$ & 0.006 \\
\hline Piperacillin-tazobactam & $54(45.3)$ & $4(20.0)$ & $50(50.5)$ & 0.01 \\
\hline Minocycline & 11(9.2) & 0 & 11(11.1) & 0.118 \\
\hline Piperacillin & $85(71.4)$ & $9(45.0)$ & $76(76.8)$ & 0.004 \\
\hline Tigecycline & $1(0.8)$ & 0 & $1(1.0)$ & 0.652 \\
\hline Ceftazidime - avibatan & 11(9.2) & $1(5.0)$ & $10(10.1)$ & 0.473 \\
\hline CRKP & $49(41.2)$ & $4(20.0)$ & $45(45.4)$ & 0.03 \\
\hline ESBL & $24(20.2)$ & $4(20.0)$ & $20(20.2)$ & 0.98 \\
\hline
\end{tabular}

Table 3 shows how T6SS positive and T6SS negative strains differ in terms of capsular serotypes and virulence characteristics. K1 and K2 capsular serotypes accounted for 10.9\% (13/119) and 7.5\% (9/119) of all $K$. pneumoniae, respectively. T6SS + strains displayed considerably greater prevalences of the K1 serotype ( $25.0 \%$ vs $8.1 \%, P=0.027)$, aerobactin ( $55.0 \%$ vs $17.2 \%, P 0.001)$, and iroB ( $45.0 \%$ vs $18.2 \%, P$ 
$=0.009)$ than T6SS + strains. T6SS-positive strains had a considerably greater incidence of hvkp than T6SS-negative strains $(50.0 \%$ vs $16.2 \%, P=0.001)$.

Table 3

Capsular serotypes and virulence factors between T6SS-positive and T6SS-negative K. pneumoniae bloodstream isolates

\begin{tabular}{|lllll|}
\hline Vairable, $\mathbf{n}(\%)$ & Total $\mathbf{n}=\mathbf{1 1 9}$ & T6SS-positive $(\mathbf{n = 2 0 )}$ & T6SS-negative $(\mathbf{n}=\mathbf{9 9})$ & $\boldsymbol{p}$-Value \\
\hline Capsular serotype & & & & \\
\hline K1 & $13(10.9)$ & $5(25.0)$ & $8(8.1)$ & 0.027 \\
\hline K2 & $9(7.5)$ & $1(5.0)$ & $8(8.1)$ & 0.653 \\
\hline Non K1/K2 & $97(81.5)$ & $14(70.0)$ & $83(83.8)$ & 0.146 \\
\hline Virulence genes & & & & 0.153 \\
\hline rmpA & $60(50.4)$ & $13(65.0)$ & $47(47.5)$ & 0.307 \\
\hline rmpA2 & $54(45.3)$ & $12(60.0)$ & $42(42.4)$ & $<0.001$ \\
\hline aerobactin & $28(23.5)$ & $11(55.0)$ & $17(17.2)$ & 0.009 \\
\hline iroB & $27(22.6)$ & $9(45.0)$ & $18(18.2)$ & 0.001 \\
\hline hvkp & $26(21.8)$ & $10(50.0)$ & $16(16.2)$ & \\
\hline
\end{tabular}

\subsection{Crude Impact of TTSS Genotype in Mortality}

Table 1 shows that the overall 30-day mortality rate was $32.8 \%$ (39 patients), with $66.7 \%$ (26 patients) dying within the first seven days of being admitted to the hospital. There were no significant variations in early (7-day) mortality according to the T6SS genotype. Late mortality among patients infected with T6SS + isolates, on the other hand, was $10 \%$ compared to $37.4 \%$ among patients infected with T6SS strains $(P=0.01)$.

The survival curve (Fig. 1A) revealed no significant differences in the 7-day cumulative likelihood of mortality between T6SS-positive and T6SS-negative KP-BSI isolates (log-rank $\mathrm{P}=.46$ ). Meanwhile, there was no significant difference in cumulative survival until day 30 (Fig. 1B) between the KP episodes that were positive or negative for T6SS (log-rank $\mathrm{P}=.38$ ).

\subsection{Biofilm formation, competitive index, and serum resistance}

T6SS + and T6SS - strains had their relative biofilm-forming activity calculated, and individual isolates were investigated in triplicate. T6SS-positive KP isolates' mean OD600 value was not substantially different from T6SS-negative isolates (mean \pm standard deviation [SD], $0.059 \pm 0.005$ vs. $0.08 \pm 0.04 ; \mathrm{P}=$ 0.303) (Fig. 2A). After $3 \mathrm{~h}$ of incubation in serum, T6SS-positive isolates revealed no significant changes 
in survival rate compared to T6SS-negative isolates (40.95\% vs. $21.73 \%$; $\mathrm{P}=0.35)$ (Fig. 2B). In vitro competition experiments were also carried out to calculate the $\mathrm{Cl}$ to see if T6SS + isolates could outcompete other bacteria. The result shown that T6SS-positive isolates were more competitive during joint incubation than T6SS-negative isolates (Fig. 2C).

\section{Discussion}

Klebsiella. pneumoniae is a common nosocomial pathogen that is causing widespread concern due to multidrug resistance and the recent emergence of hypervirulent strains in clinical settings. Capsule, Pili, lipopolysaccharide (LPS), and iron carriers are now known to be pathogenic factors [29]. RmpA is a plasmid-based virulence element in K. pneumoniae that regulates capsular polysaccharide production [30]. Enterobactin, yersiniabactin, salmochelin, and aerobactin are the four iron-absorbing molecules (iron carriers) found in K. pneumoniae[31]. Aerobactin is thought to be the primary virulence factor of hvKP since it is involved in iron transport, proliferation, and virulence in K. pneumoniae [32]. String test in combination with aerobactin and the rmpA index improved the detection rate of hvKP, according to Li et al.

In this investigation, strains positive for rmpA and aerobactin were classified as hvKP, and the results revealed that $26(21.8 \%)$ of the strains were hvKP. T6SSpositive strains were defined as those that were positive for icmF, vgrG, and hcp in a prior investigation. Based on these criteria, our research found that T6SS genes were present in $16.8 \%$ of $K$. pneumoniae bloodstream isolates. However, only patients with bloodstream infections were included in this investigation, not individuals with chronic infections who might have been colonized by mature K. pneumoniae colonies. T6SS positive strains had a greater detection rate of $\mathrm{K} 1$ capsular serotypes and four virulence genes than T6SS negative bacteria, according to the findings. The incidence of hvkp was substantially greater in T6SS-positive strains in comparison to the T6SS-negative strains. This research backed up the pathogenicity of the T6SS-positive KP strains.

Antimicrobial sensitivity was found in hypervirulent bacteria in previous research. T6SS-positive $K$. pneumoniae strains had lower antimicrobial resistance rates than T6SS-negative bacteria in this investigation. With the exception of minocycline, tigecycline, and ceftazidime averbactam, practically all antibiotic drugs had significantly greater resistance rates for T6SS negatives than for T6SS positives. The percentage of multidrug-resistant, carbapenem-resistant, and ESBL-producing K. pneumoniae, on the other hand, was extremely high. Multidrug resistance levels linked with this species have skyrocketed, posing a global threat, in particular for carbapenemase-producing K. pneumoniae. The antibacterial activity of T6SS in K. pneumoniae, on the other hand, is little understood. A study found that -lactam antibiotics, at a sub-inhibitory concentration increased T6SS-dependent killing by stimulating the production and secretion of the CRKP HS11286 T6SS. As a result of the antibiotic stress, CRKP HS1186 outperforms the T6SS/multidrug-resistant strain in terms of growth superiority.

We investigated the risk variables impacting the prognosis of $K$. pneumoniae BSI in this study, which included a complete clinical and microbiological evaluation. The significant death rate associated with this infection is confirmed by our findings, with $21.8 \%$ and $32.8 \%$ of patients dying within the first 7 and 
30 days, respectively. This discovery is in line with recent findings indicating that this condition is much more fatal than bacteremia produced by other germs.

T6SS+ isolates had stronger biofilm-forming activity and survived longer when present in normal human serum than T6SS- isolates, according to previous research. However, the findings of our study on $K$. pneumoniae isolates contradict this concept. The differences between $K$. pneumoniae and other bacteria could be due to species-specific characteristics. It's unclear whether T6SS+ isolates' biofilm-forming ability and serum resistance are related to the T6SS's own function. A number of investigations have shown that the T6SS is involved in biofilm production in a variety of bacteria; however, certain bacteria do not need the T6SS to create biofilms. Type 3 pili and capsular polysaccharides (CPs) are the most critical surface features of $K$. pneumoniae that take part in the production process [34-35]. Pili mediate long-term adherence, whereas CPs influence intercellular communication and biofilm structure in the end. The function of biofilm can also be affected by mutations in certain K. pneumoniae genes [36].

In this investigation, we discovered that T6SS+ isolates outperformed T6SS-isolates in terms of outcompeting E. coli. In a vast majority of T6SS-carrying bacteria, the significance of the T6SS in intraspecies and interspecies bacterial competition has been recognized as a key characteristic of the system. The T6SS has been shown to direct effector proteins to other bacteria of the likes of $P$. aeruginosa, $V$. cholerae, and $S$. marcescens, allowing the organism to get involved in competition more successfully against other bacteria in its growing environment [37-40]. Nevertheless, the antibacterial properties of the T6SS in K. pneumoniae have yet to be discovered. The T6SS renders a fitness advantage for T6SS+ clinical isolates of $K$. pneumoniae, according to our findings.

\section{Conclusion}

T6SS-positive K. pneumoniae was common in people who had BSIs. T6SS-positive K. pneumoniae strains apparently looked hypervirulent. In comparison to T6SS-negative isolates, $K$. pneumoniae isolates with T6SS-positive might outcompete Escherichia coli. T6SS+ isolates, on the other hand, did not show stronger biofilm-forming activity or a higher survival rate in the presence of normal human serum in comparison to T6SS- isolates

\section{Abbreviations}

T6SS Type VI secretion system

BSI Bloodstream infection

Hcp Hemolysin-coregulated protein

VgrG Valine-glycine repeat protein G

IcmF lintracellular multiplication protein $\mathrm{F}$ 
TssM T6SS inner membrane protein

MDR Multi-drug resistance

TTP Time to positive

PBS Phosphate-buffered saline

NHS Normal human serum

CFUs Colony Forming Units

IQR Interquartile ranges

$\mathrm{CCl}$ Charlson Comorbidity Index

CRKP Carbapenem-resistant Klebsiella. Pneumoniae

LPS lipopolysaccharide

\section{Declarations}

\section{Acknowledgements}

We thank the First Affiliated Hospital of Anhui Medical University for their cooperation.

\section{Author Contributions}

Investigation, Yin Zhang and Ying Huang; Project administration, Ying Huang; Writing - original draft, Yin Zhang.

\section{Availability of data and materials}

All data generated or analyzed during this study are included in this manuscript.

\section{Ethics approval and consent to participate}

The study was approved by the Medical Ethics Committee of the First Affiliated Hospital of Anhui Medical University. Written informed consent was waived by the Medical Ethics Committee of the First Affiliated Hospital of Anhui Medical University due to the anonymized retrospective nature of the analysis.

\section{Funding}

This research did not receive any specific grant from funding agencies in the public, commercial, or notfor-profit sectors. 
Not applicable.

\section{Declaration of Competing Interest}

None.

\section{References}

1. Ko WC, Paterson DL, Sagnimeni AJ, Hansen DS, Von Gottberg A, Mohapatra S, Casellas JM, Goossens H, Mulazimoglu L, Trenholme G, Klugman KP, McCormack JG, Yu VL. Community-acquired Klebsiella pneumoniae bacteremia: global differences in clinical patterns. Emerg Infect Dis. 2002 Feb;8(2):160-6.

2. Lederman ER, Crum NF. Pyogenic liver abscess with a focus on Klebsiella pneumoniae as a primary pathogen: an emerging disease with unique clinical characteristics. Am J Gastroenterol. 2005 Feb;100(2):322-31.

3. Shon AS, Bajwa RP, Russo TA. Hypervirulent (hypermucoviscous) Klebsiella pneumoniae: a new and dangerous breed. Virulence. 2013 Feb 15;4(2):107-18.

4. Russo TA, Marr CM. Hypervirulent Klebsiella pneumoniae. Clin Microbiol Rev. 2019 May 15;32(3):e00001-19.

5. Wang X, Xie Y, Li G, Liu J, Li X, Tian L, Sun J, Ou HY, Qu H. Whole-Genome-Sequencing characterization of bloodstream infection-causing hypervirulent Klebsiella pneumoniae of capsular serotype K2 and ST374. Virulence. 2018;9(1):510-21.

6. Liu C, Guo J. Hypervirulent Klebsiella pneumoniae (hypermucoviscous and aerobactin positive) infection over 6 years in the elderly in China: antimicrobial resistance patterns, molecular epidemiology and risk factor. Ann Clin Microbiol Antimicrob. 2019;18(1):4.

7. Basler M, Pilhofer M, Henderson GP, Jensen GJ, Mekalanos JJ. Type VI secretion requires a dynamic contractile phage tail-like structure. Nature. 2012 Feb 26;483(7388):182-6.

8. Ho BT, Dong TG, Mekalanos JJ. A view to a kill: the bacterial type VI secretion system. Cell Host Microbe. 2014;15(1):9-21.

9. Kudryashev M, Wang RY, Brackmann M, Scherer S, Maier T, Baker D, DiMaio F, Stahlberg H, Egelman $\mathrm{EH}$, Basler M. Structure of the type VI secretion system contractile sheath. Cell. 2015 Feb 26;160(5):952-962.

10. Silverman JM, Agnello DM, Zheng H, Andrews BT, Li M, Catalano CE, Gonen T, Mougous JD. Haemolysin coregulated protein is an exported receptor and chaperone of type $\mathrm{VI}$ secretion substrates. Mol Cell. 2013 Sep 12;51(5):584 - 93.

11. Pukatzki S, Ma AT, Sturtevant D, Krastins B, Sarracino D, Nelson WC, Heidelberg JF, Mekalanos JJ. Identification of a conserved bacterial protein secretion system in Vibrio cholerae using the Dictyostelium host model system. Proc Natl Acad Sci U S A. 2006 Jan;31(5):1528-33. 103(. 
12. Monjarás Feria J, Valvano MA. An Overview of Anti-Eukaryotic T6SS Effectors. Front Cell Infect Microbiol. 2020 Oct 19;10:584751.

13. Ma LS, Narberhaus F, Lai EM. IcmF family protein TssM exhibits ATPase activity and energizes type VI secretion. J Biol Chem. 2012;287(19):15610-21.

14. Bönemann G, Pietrosiuk A, Diemand A, Zentgraf $H$, Mogk A. Remodelling of VipA/VipB tubules by ClpV-mediated threading is crucial for type VI protein secretion. EMBO J. 2009 Feb 18;28(4):315-25.

15. Pietrosiuk A, Lenherr ED, Falk S, Bönemann G, Kopp J, Zentgraf H, Sinning I, Mogk A. Molecular basis for the unique role of the AAA + chaperone ClpV in type VI protein secretion. J Biol Chem. 2011 Aug 26;286(34):30010-21.

16. Basler M, Pilhofer M, Henderson GP, Jensen GJ, Mekalanos JJ. Type VI secretion requires a dynamic contractile phage tail-like structure. Nature. 2012 Feb 26;483(7388):182-6.

17. Ma LS, Lin JS, Lai EM. An Icm F family protein,Imp LM,is an integral inner membrane protein interacting with Imp KL,and its walker a motif is required for type $\mathrm{VI}$ secretion system-mediated Hcp secretion in Agrobacterium tumefaciens.Journal of Bacteriology,2009,191(13):4316-4329.

18. Ma LS, Narberhaus F, Lai EM. IcmF family protein TssM exhibits ATPase activity and energizes type VI secretion. J Biol Chem. 2012;287(19):15610-21.

19. Murdoch SL, Trunk K, English G, Fritsch MJ, Pourkarimi E, Coulthurst SJ. The opportunistic pathogen Serratia marcescens utilizes type VI secretion to target bacterial competitors. J Bacteriol. 2011 Nov;193(21):6057-69.

20. Gallique M, Decoin V, Barbey C, Rosay T, Feuilloley MG, Orange N, Merieau A. Contribution of the Pseudomonas fluorescens MFE01 Type VI Secretion System to Biofilm Formation. PLoS One. 2017 Jan 23;12(1):e0170770.

21. Decoin V, Gallique M, Barbey C, Le Mauff F, Poc CD, Feuilloley MG, Orange N, Merieau A. A Pseudomonas fluorescens type 6 secretion system is related to mucoidy, motility and bacterial competition. BMC Microbiol. 2015 Mar;26:15:72.

22. Spiewak HL, Shastri S, Zhang L, et al. Burkholderia cenocepacia utilizes a type VI secretion system for bacterial competition [published online ahead of print, 2019 Jan 9]. Microbiologyopen. 2019;8(7):e774.

23. Ning Y, Hu R, Yao G, Bo S. Time to positivity of blood culture and its prognostic value in bloodstream infection. Eur J Clin Microbiol Infect Dis. 2016 Apr;35(4):619-24.

24. Cunha BA. Sepsis and septic shock: selection of empiric antimicrobial therapy. Crit Care Clin. 2008 Apr;24(2):313-34, ix.

25. Merritt JH, Kadouri DE, O'Toole GA. Growing and analyzing static biofilms. Curr Protoc Microbiol. 2005 Jul;Chap. 1:Unit 1B.1.

26. Juyoun, Shin. Kwan Soo Ko. Effect of plasmids harbouring blaCTX-M on the virulence and fitness of Escherichia coli ST131 isolates. International Journal of Antimicrobial Agents Volume. 2015;46(2):214-8. 
27. Spiewak HL, Shastri S, Zhang L, et al. Burkholderia cenocepacia utilizes a type VI secretion system for bacterial competition [published online ahead of print, 2019 Jan 9]. Microbiologyopen. 2019;8(7):e774.

28. Weber BS, Miyata ST, Iwashkiw JA, et al. Genomic and functional analysis of the type VI secretion system in Acinetobacter. PLoS One. 2013;8(1):e55142.

29. Li B, Zhao Y, Liu C. Zhenhong Chen, and Dongsheng Zhou. Molecular pathogenesis of Klebsiella pneumoniae. Future Microbiology 2014 9:9, 1071-1081.

30. Hsu CR, Lin TL, Chen YC, Chou HC, Wang JT. The role of Klebsiella pneumoniae rmpA in capsular polysaccharide synthesis and virulence revisited. Microbiology. 2011;157(Pt 12):3446-57.

31. Russo TA, Shon AS, Beanan JM, et al. Hypervirulent $K$. pneumoniae secretes more and more active iron-acquisition molecules than 'classical' $K$. pneumoniae thereby enhancing its virulence. PLoS ONE. 2011;6(10):e26734.

32. Russo TA, Olson R, Fang CT, et al. Identification of Biomarkers for Differentiation of Hypervirulent Klebsiella pneumoniae from Classical K. pneumoniae. J Clin Microbiol. 2018;56(9):e00776-18.

33. Liu L, Ye M, Li X, Li J, Deng Z, Yao YF, Ou HY. Identification and Characterization of an Antibacterial Type VI Secretion System in the Carbapenem-Resistant Strain Klebsiella pneumoniae HS11286. Front Cell Infect Microbiol. 2017;7:442.

34. de Oliveira Júnior NG, Franco OL. Promising strategies for future treatment of Klebsiella pneumoniae biofilms. Future Microbiol. 2020 Jan;15:63-79.

35. Clegg S, Murphy CN. Epidemiology and Virulence of Klebsiella pneumoniae. Microbiol Spectr. 2016 Feb;4(1).

36. Mostafavi M, Wang L, Xie L, Takeoka KT, Richie DL, Casey F, Ruzin A, Sawyer WS, Rath CM, Wei JR, Dean CR. Interplay of Klebsiella pneumoniae fabZ and IpxC Mutations Leads to LpxC InhibitorDependent Growth Resulting from Loss of Membrane Homeostasis. mSphere. 2018 Oct 31;3(5):e00508-18.

37. Hood RD, Singh P, Hsu F, Güvener T, Carl MA, Trinidad RR, Silverman JM, Ohlson BB, Hicks KG, Plemel RL, Li M, Schwarz S, Wang WY, Merz AJ, Goodlett DR, Mougous JD. A type VI secretion system of Pseudomonas aeruginosa targets a toxin to bacteria. Cell Host Microbe. 2010 Jan 21;7(1):25-37.

38. Maclntyre DL, Miyata ST, Kitaoka M, Pukatzki S. The Vibrio cholerae type VI secretion system displays antimicrobial properties. Proc Natl Acad Sci U S A. 2010 Nov 9;107(45):19520-4.

39. Murdoch SL, Trunk K, English G, Fritsch MJ, Pourkarimi E, Coulthurst SJ. The opportunistic pathogen Serratia marcescens utilizes type VI secretion to target bacterial competitors. J Bacteriol. 2011 Nov;193(21):6057-69.

40. Spiewak HL, Shastri S, Zhang L, et al. Burkholderia cenocepacia utilizes a type VI secretion system for bacterial competition [published online ahead of print, 2019 Jan 9]. Microbiologyopen. 2019;8(7):e774.

\section{Figures}



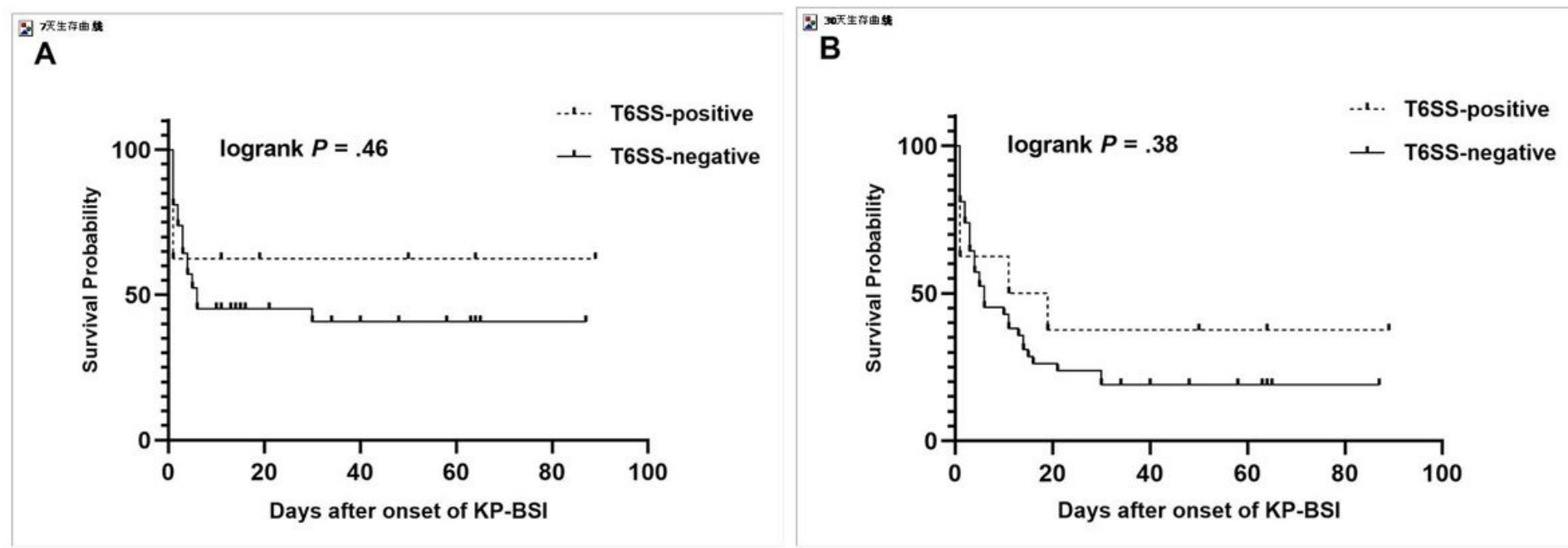

\section{Figure 1}

(A) Probability estimate for 7-day mortality of K. pneumoniae BSI according to T6SS genotype. (B) Probability estimate for 30 -day mortality of K. pneumoniae BSI according to T6SS genotype. 


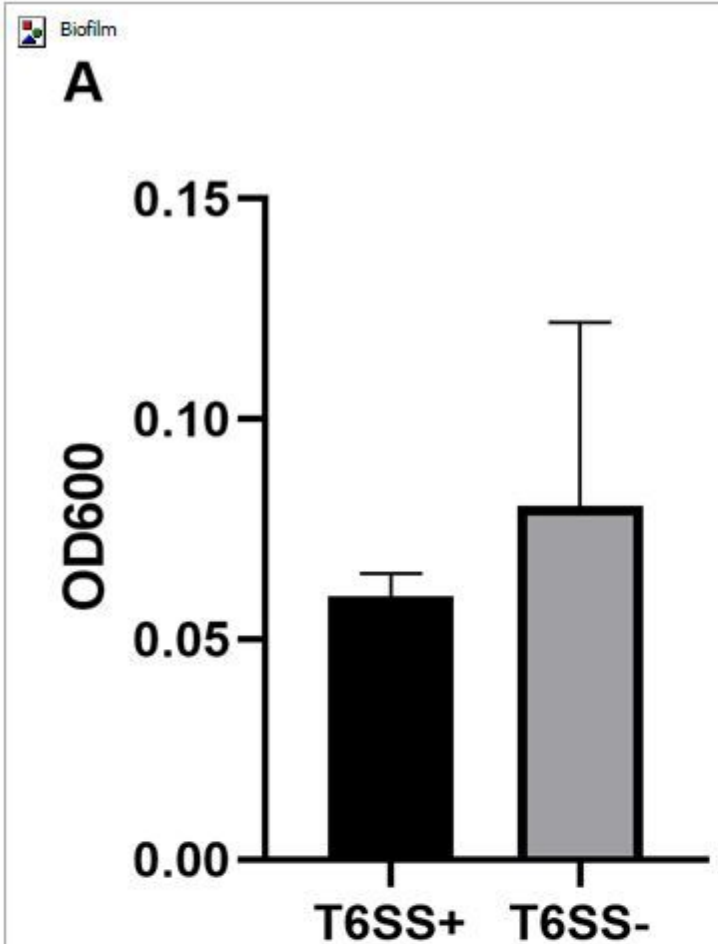

B
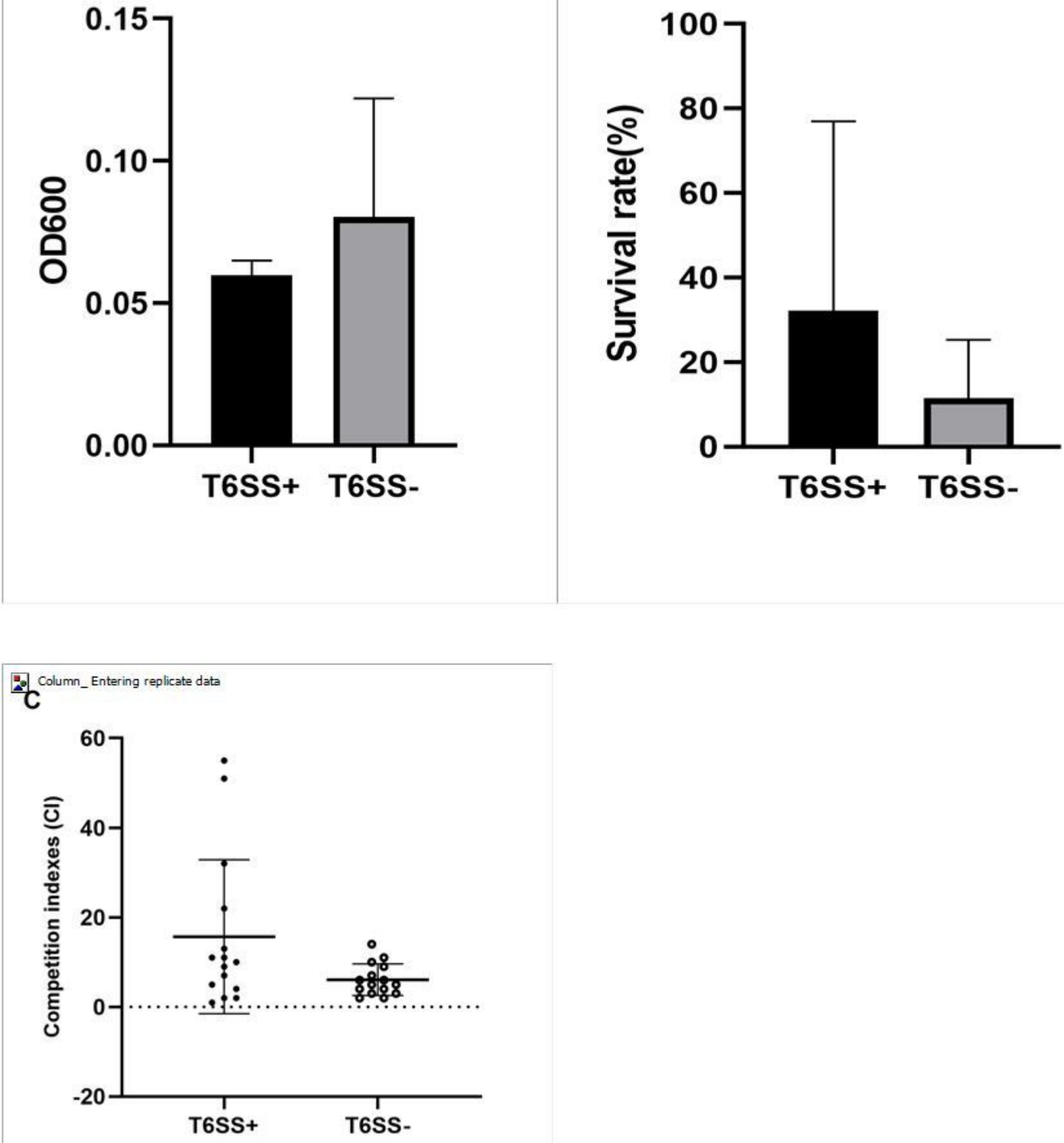

Figure 2

(A) Mean \pm standard deviation optical density at $600 \mathrm{~nm}$ of T6SS-positive and T6SS-negative klebsiella pneumoniae isolates stained with crystal violet in the biofilm formation assay. (B) Serum resistance assay for all T6SS-positive and T6SS-negative K. pneumoniae isolates. Serum resistance as a viability ratio (colony forming units [CFUs] of serum-bacterial suspension/ CFUs of bacterial suspension without normal human serum). (C) In vitro competitive index (Cl) values of T6SS K. pneumoniae isolates. Each 
circle represents one of the $\mathrm{Cl}$ values obtained from the number of ampicillin-resistant CFUs (K. pneumoniae) divided by the number of ampicillin-susceptible CFUs (E. coli). 